\title{
Introduction
}

\section{Basic Biomechanics}

\author{
G.J. Verkerke ${ }^{\mathrm{a}, \mathrm{b}, *}$ and T.C. Lee ${ }^{\mathrm{c}}$ \\ ${ }^{a}$ Department of Biomedical Engineering, University Medical Center Groningen, University of \\ Groningen, Groningen, The Netherlands \\ ${ }^{\mathrm{b}}$ Department of Biomechanical Engineering, University of Twente, Enschede, The Netherlands \\ ${ }^{\mathrm{c}}$ Department of Anatomy, Royal College of Surgeons in Ireland, Dublin, Ireland
}

\section{Introduction}

Biomechanics is the science that examines forces acting upon a biological structure and the resulting effects - deformation and acceleration.

We start with the last two, because they are easy to define:

- Acceleration is defined as a change in velocity or speed.

- Deformation is defined as a change in shape and/or size of an object.

A force is more difficult to define, because it is an abstract term; forces cannot be seen. We know that gravity forces, magnetic forces, spring forces, etc. exist, because we can observe and feel their effect. So to define a force we could use the effect of a force:

When an object starts to move (accelerate) or change its velocity or direction of movement or when an object is deformed, a force is acting upon it.

Biomechanics provides basic knowledge in the field of Biomedical Engineering. It is applied in almost all medical disciplines, and especially in orthopaedics, cardiology, rehabilitation, physical and occupational therapy, sports medicine and ergonomics.

Biomechanics is a specialisation of mechanics. It observes the effects of forces acting upon living objects or produced by living objects. Mechanics observes the effects of forces on dead, technical objects and is the oldest a branch of physics.

Archimedes (287-212 BC) described the functioning of a lever (Fig. 1a). The pulley, inclined plane, and wrench were also described and used in ancient times, as can be seen in Greek and Roman buildings and machines. Galileo Galilei (1564-1642) studied dynamics, Isaac Newton (1642-1727) described

\footnotetext{
*Address for correspondence: G.J. Verkerke, Department of Biomedical Engineering, University Medical Center Groningen, University of Groningen, Groningen, The Netherlands. Tel.: +31 50 3633126; Fax: +31 50 3633139; E-mail: g.j.verkerke@med.umcg.nl.
} 
(a)

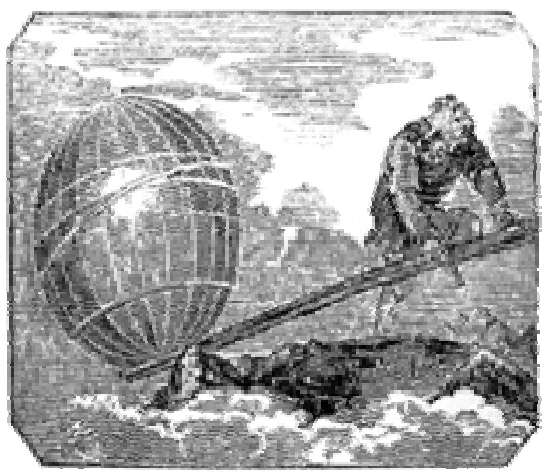

(b)

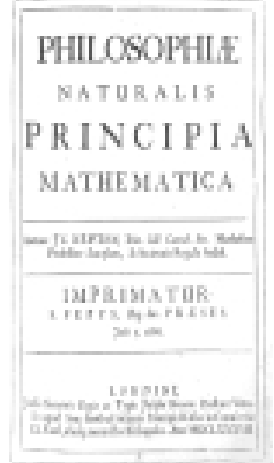

Fig. 1. (a) 'Give me a place to stand on, and I will move the earth', a quote of Archimedes in relation to the lever. (b) Isaac Newton: Philosophiae Naturalis Principia Mathematica, 1687.

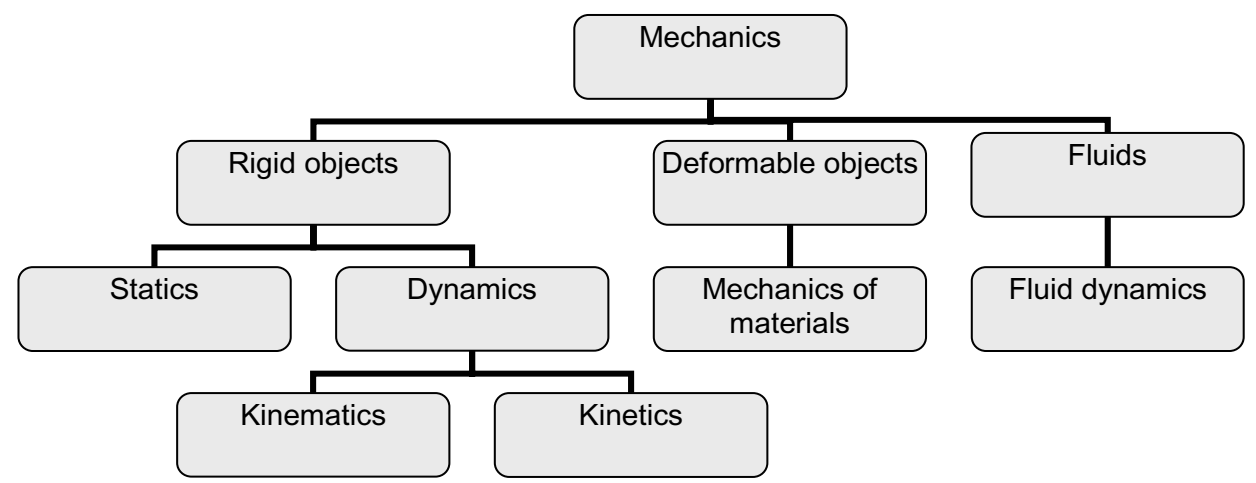

Fig. 2. The different branches of mechanics and the nature of the objects they deal with.

three fundamental laws of mechanics, after he was hit by an apple (Fig. 1b). The First Law states that, if an object is not subjected to a force, this object will not start to move or change its velocity. The Second Law states that, if a body is subjected to a force, it will accelerate (change its velocity or start moving). The Third Law states that, if you apply a force to an object, this object will exert the same force, in the opposite direction. If you are sitting on a chair, you exert a gravity force on this chair. The chair, on its turn, applies an equal force on you. If you don't believe that, put a thumb tack on the chair before sitting on it and you will experience the force of the chair acting on you!

Mechanics can be subdivided into several areas (Fig. 2). First by the nature of the object, a solid or a fluid, then solid objects can be deformable or rigid; in a rigid object deformations are not considered to happen. Statics is the field of mechanics that studies rigid objects that don't move, so the forces acting upon them balance each other. Mechanics of materials studies the deformation of objects that don't move. Dynamics studies rigid objects that move. Kinematics is a special branch of dynamics that only studies movement, while kinetics studies the forces acting upon objects and the resulting movements.

We now discover an essential condition for studying mechanics, we have to model reality. Reality is too complex to analyse, so we have to simplify it by making many assumptions: We assume that for studying statics and dynamics the deformation of objects is not important. The most difficult part of studying mechanics is to make an appropriate model that is simple enough to allow the application of the formulae, but complex enough to predict the proper effects of forces on an object. 
Here we will present the formulae you can use to predict the effect of forces, but we will also discuss how to make a good model.

This chapter is divided into four sections dealing with statics, dynamics of solids, mechanics of materials and fluid dynamics (Fig. 2). 